\title{
Nonequilibrium steady states
}

\author{
VoJKan JAŠIĆ ${ }^{1}$, Claude-Alain PILlet ${ }^{2}$ \\ ${ }^{1}$ Department of Mathematics and Statistics \\ McGill University \\ 805 Sherbrooke Street West \\ Montreal, QC, H3A 2K6, Canada \\ jaksic@math.mcgill.ca
}

\author{
${ }^{2}$ CPT-CNRS, UMR 6207 \\ Université du Sud Toulon-Var \\ B.P. 20132 \\ 83957 La Garde Cedex, France \\ pilleteuniv-tln.fr
}

Equilibrium states are the central objects of equilibrium statistical mechanics. To a large extent the success of this theory is due to the fact that the equilibrium states of a system can be constructed and characterized without explicit reference to the dynamics of this system. This is a truly amazing fact because the concept of equilibrium state and the ultimate justification of the whole theory, as formulated by Boltzmann and its followers, do depend on the dynamics.

Nonequilibrium statistical mechanics has a completely different status. It is a more difficult theory, both conceptually and technically, because a deep control of the dynamics is required to understand even the most basic nonequilibrium properties of the system. The simplest conceivable elements of the large manifold of nonequilibrium states of a system are the steady states in which it settles under the action of weak, stationary external forces. Such a forcing can be achieved either by applying an external field or by imposing a sustained gradient of intensive thermodynamic parameters (e.g., a constant temperature drop) across the system. Even though definite progresses have been made in the recent years we are still far from a coherent theory of steady states if such a theory exists at all.

\section{Phenomenological theory and linear response}

To appreciate recent rigorous results in the field a basic knowledge of nonequilibrium thermodynamics is required. This section is a condensed introduction to the phenomenological theory of nonequilibrium steady states (NESS). The interested reader should consult $[\mathrm{dGM}]$ or $[\mathrm{C}]$ for detailed expositions.

Consider bringing into contact two systems $\mathcal{S}_{1}, \mathcal{S}_{2}$, each of which is in thermal equilibrium. Denote by $S_{\alpha}\left(A_{\alpha 1}, A_{\alpha 2}, \ldots\right)$ the entropy of $\mathcal{S}_{\alpha}$ as a function of its extensive thermodynamic parameters. The combined system $\mathcal{S}_{1}+\mathcal{S}_{2}$ being otherwise isolated, the values of $A_{j}=A_{1 j}+A_{2 j}$ are fixed and the entropy of the joint system is given by $S=\sum_{\alpha} S_{\alpha}\left(A_{\alpha 1}, A_{\alpha 2}, \ldots\right)$. The conditions for joint thermal equilibrium are thus

$$
X_{j}=\frac{\partial S}{\partial A_{1 j}}=\frac{\partial S_{1}}{\partial A_{1 j}}-\frac{\partial S_{2}}{\partial A_{2 j}}=I_{1 j}-I_{2 j}=0,
$$

where $I_{\alpha j}$ denotes the intensive thermodynamic parameter conjugate to $A_{\alpha j}$. If the combined system is not in thermal equilibrium, then the $X_{j} \neq 0$ act as thermodynamic forces. Their effect is to generate fluxes of the extensive quantities which can be measured by

$$
\Phi_{j}=\frac{\mathrm{d} A_{1 j}}{\mathrm{~d} t}
$$

The rate of entropy production is then

$$
\frac{\mathrm{d} S}{\mathrm{~d} t}=\sum_{j} \frac{\partial S}{\partial A_{1 j}} \frac{\mathrm{d} A_{1 j}}{\mathrm{~d} t}=\sum_{j} X_{j} \Phi_{j} .
$$


Near equilibrium the forces $X_{j}$ are weak and first order perturbation theory in these forces - also called linear response theory - becomes a good approximation. Writing the fluxes as

$$
\Phi_{j}=\sum_{k} L_{k j} X_{k}+\text { higher order terms },
$$

defines the kinetic or transport coefficients $L_{k j}$. The matrix $L=\left(L_{k j}\right)$ is called Onsager matrix. It depends only on the intensive parameters $I_{1 j}$. One of the most basic problem of nonequilibrium statistical mechanics is the calculation of Onsager's matrix starting from a microscopic description of the system.

In linear response theory the entropy production rate becomes

$$
\frac{\mathrm{d} S}{\mathrm{~d} t} \simeq \sum_{k} L_{k j} X_{k} X_{j} .
$$

Thus, the second law of thermodynamics implies that the symmetric part of $L$ is positive. Moreover, if the systems are time-reversal invariant then the Onsager reciprocity relations

$$
L_{k j}=L_{j k},
$$

hold, i.e., the matrix $L$ is symmetric. If the systems are not time reversal invariant, these relations have to be modified consequently. For example if an external magnetic field $B$ is applied then the matrix $L$ depends parametrically on $B$ and the Onsager-Casimir relations

$$
L_{k j}(B)=L_{j k}(-B),
$$

hold.

Remarks. 1. Applying the above discussion to small volume elements of a macroscopic body it is possible to obtain a phenomenological description of the local structure of a nonequilibrium state under the so called local thermodynamic equilibrium (LTE) hypothesis. The reader should consult the above references for details.

2. Some variational characterizations of NESS have been proposed, most notably the principle of minimal entropy production. However the status of such principles is still controversial and their validity seems to be only approximate and limited to some special systems. See the [Variational principle for NESS] and [MN] for more information on this subject.

\section{Microscopic theory}

A microscopic theory of NESS must be based on the asymptotic analysis of the dynamics which describes the evolution of the system. A non-isolated system is driven out of equilibrium by the forces exerted by its environment. Under appropriate conditions the system eventually settles in a steady state. Denote by $\langle\cdot\rangle_{0}$ the initial state, in the sense of statistical mechanics, of the system and its environment. If $t \mapsto\langle\cdot\rangle_{t}$ denotes the evolution of this state then the limit

$$
\langle\cdot\rangle_{+}=\lim _{t \rightarrow \infty}\langle\cdot\rangle_{t},
$$

or more generally

$$
\langle\cdot\rangle_{+}=\lim _{t \rightarrow \infty} \frac{1}{t} \int_{0}^{t}\langle\cdot\rangle_{s} \mathrm{~d} s,
$$

defines a stationary state.

On physical grounds one expects more. Namely the limiting state $\langle\cdot\rangle_{+}$should be invariant under local changes of the initial state $\langle\cdot\rangle_{0}$. To elaborate on this essential point let us consider a smooth dynamical system on a compact phase space $X \subset \mathbb{R}^{n}$. A physically natural statistics of the configurations of the system is provided by 
the normalized Lebesgue measure $\mathrm{d} x$ on $X$ : Initial configurations sampled according to $\mathrm{d} x$ can be considered typical. This has nothing to do with dynamics and any distribution $\rho(x) \mathrm{d} x$ with strictly positive density $\rho$ would serve the same purpose. Accordingly, we expect all these initial states to yield the same limiting state $\langle\cdot\rangle_{+}$. For systems with infinitely many degrees of freedom there is nothing like Lebesgue measure and we must provide a reference state to sample the initial configurations. The purpose of this state is to specify the thermodynamic state of the system. Suppose for example that the system is driven out of equilibrium by several infinite reservoirs. Specifying an equilibrium state (a Gibbs measure or a KMS state) for each reservoir sets the temperature and other relevant thermodynamic parameters of each reservoir. States with different thermodynamic parameter are mutually singular and will lead to different NESS. A contrario, local perturbations of these equilibrium states will relax to equilibrium under the reservoir dynamics. Therefore, as in the finite dimensional case, we expect the limiting state $\langle\cdot\rangle_{+}$to be insensitive to such local perturbations. In the language of $C^{*}$-dynamical systems, the NESS should only depends on the folium of the initial state.

The minimal goal of a microscopic theory of NESS is a mathematical derivation of the main results of linear response theory: Onsager reciprocity relations and fluctuation-dissipation relations (e.g. the Green-Kubo formula for the kinetic coefficients $L_{k j}$, see [Linear response theory]). A more ambitious program is to explore the largely unknown fields of far from equilibrium steady states thermodynamics.

Discarding the obvious distinction between classical and quantum systems, recent progresses in these directions can be classified in two categories according to the level of description of microscopic dynamics: Hamiltonian or Markovian. The first category is of course more fundamental since microscopic dynamics is inherently Hamiltonian. However, the facts that

- various roads (scaling limits, coarse graining, restriction to specific degrees of freedom, etc...) lead from Hamiltonian dynamics to Markov processes;

- Hamiltonian dynamics is much more difficult to control than Markovian evolution;

explain why most available results belong to the second category. In fact the Markovian nature of time evolution was already at the heart of the pioneering works of Onsager and his followers (see [dGM]).

A large body of works have been devoted to the study of interacting particle systems. This class of Markovian models is discussed in details in [Interacting particle systems], [Hydrodynamic scaling limit], [Macroscopic equations in equilibrium and nonequilibrium statistical mechanics], [Nonequilibrium stationary states (stochastic)], see also [KL], [BDGJ]. We shall not consider such models here but restrict our attention to a small mechanical system $\mathcal{S}$, with a finite number of degrees of freedom, driven out of equilibrium by some external forcing.

In the framework of classical mechanics, two approaches are possible. In what we will call the canonical approach the system $\mathcal{S}$ interacts with infinitely extended reservoirs $\mathcal{R}_{1}, \mathcal{R}_{2} \ldots$ Apart from its physical appeal, the advantage of the canonical approach is that it allows for a Hamiltonian description of the coupled system $\mathcal{S}+\mathcal{R}_{1}+\cdots$. The price to pay is the necessity to deal with infinitely many degrees of freedom. As a result only very simple reservoirs (e.g., ideal gases or free fields) can be considered. Fortunately these simple systems also do a good job as reservoirs in the sense that, at least in dimension larger than two, the local properties of their internal state do not change too much as a result of the coupling to the system. Moreover, it is possible to design the coupling of these reservoirs to the system in such a way that the dynamics of $\mathcal{S}$ becomes essentially Markovian (see [EPR]). This is an important feature hardly achieved in other approaches (except of course in an axiomatic way, see below). We refer to [RB] for an introduction to recent results in the canonical approach to classical NESS.

It is important to realize that, in the canonical approach, the reservoirs serve two complementary purposes. First they have a chaotic internal dynamics. The forces they exert on the system $\mathcal{S}$ act as a source of randomness. Under the action of these forces, the dynamics of $\mathcal{S}$ becomes itself chaotic. Second, the dynamics of the reservoir is dissipative: Any local perturbation get carried away to spatial infinity. This allows the system $\mathcal{S}$ to relax from large fluctuations in its internal state by transferring energy, momentum, ..., to the reservoirs.

In the microcanonical approach the same effects of the environment on the motion of the system are obtained in a different way. Fluctuations of the system $\mathcal{S}$ are generated by a non-conservative external force. Under the action of this force the system would constantly heat-up. To avoid this effect and allow the system to settle in a 
stationary state a dissipative force is added - a so called thermostat. A popular example is the Gaussian thermostat which keep the energy (or the kinetic energy) of $\mathcal{S}$ constant. The advantage of the microcanonical approach is that it leads to a dynamical system on a finite dimensional compact manifold (a surface of constant energy of $\mathcal{S}$ ). In particular, numerical experiments are much easier to perform on such a system than on the infinite dimensional systems obtained in the canonical approach. This fact has recently led to the numerical discovery of an unexpected fluctuation relation in far from equilibrium NESS [ECM]. This relation has been turned into a mathematical statement by combining the microcanonical approach with an axiomatic setup - the so called chaotic hypothesis [GC]. We refer to [R1], [D] for detailed introductions to this approach and to the articles [NESS in classical statistical mechanics], [Gallavotti-Cohen chaotic hypothesis], [Sinai-Bowen-Ruelle distribution], [Numerical simulations in nonequilibrium statistical mechanics], [Dynamical systems approach to nonequilibrium statistical mechanics]. A proof of the fluctuation relation for the canonical NESS of an anharmonic chain of oscillators has also been obtained in $[R B T]$. More recently the fluctuation relation has been observed in real experiments in various systems of very different nature, confirming its apparent universality.

Equivalence of the equilibrium ensembles (microcanonical, canonical, grand canonical,...) is a cornerstone of equilibrium statistical mechanics (see [Equivalence of Ensembles]). It is conjectured that a similar equivalence holds for NESS. In the thermodynamic limit, i.e., as the size of $\mathcal{S}$ becomes large, one expects that the statistical properties of macroscopic observables in a NESS do not depend on the construction of the NESS. Microcanonical NESS with different kinds of thermostats as well as canonical NESS with different kinds of reservoirs should yield the same statistics. To our knowledge the only available result in this direction is [R2].

Due to the intrinsic Hamiltonian nature of quantum mechanics the canonical approach is the only way to define and study the NESS of a quantum system (at least within a purely quantum description). We refer to [NESS in quantum statistical mechanics] for a detailed discussion of quantum NESS.

\section{References}

[BDGJ] Bertini, L., De Sole, A., Gabrielli, D., Jona-Lasinio, G., Landim, C.: Large deviation approach to non equilibrium processes in stochastic lattice gases. Bull. Braz. Math. Soc. New Series 37, 1 (2006).

[C] Callen, H.B.: Thermodynamics and an Introduction to Thermostatistics. Second edition. Wiley, New York (1985).

[D] Dorfman, J.R.: An Introduction to Chaos in Nonequilibrium Statistical Mechanics. Cambrige University Press, Cambridge (1999).

[dGM] De Groot, S.R., Mazur, P.: Non-Equilibrium Thermodynamics. North-Holland, Amsterdam (1969).

[ECM] Evans, D.J., Cohen, E.G.D., Morriss, G.P.: Probability of second law violations in shearing steady states. Phys. Rev. Lett. 71, 2401 (1993).

[EPR] Eckmann, J.-P., Pillet, C.-A., Rey-Bellet, L.: Non-equilibrium statistical mechanics of anharmonic chains coupled to two heat baths at different temperatures. Commun. Math. Phys. 201, 657 (1999).

[GC] Gallavotti, G., Cohen, E.G.D.: Dynamical ensembles in nonequilibrium statistical mechanics. Phys. Rev. Lett. 74, 2694 (1995).

[KL] Kipnis, C., Landim, C.: Scaling Limits of Interacting Particle Systems. Springer, Berlin (1999).

[MN] Maes, C., Netočný, K.: Minimum entropy production principle from a dynamical fluctuation law. To appear in J. Math. Phys. (2007).

[R1] Ruelle, D.: Smooth dynamics and new theoretical ideas in nonequilibrium statistical mechanics. J. Stat. Phys. 95, 393 (1999). 
[R2] Ruelle, D.: A remark on the equivalence of isokinetic and isoenergetic thermostats in the thermodynamic limit. J. Stat. Phys. 100, 757 (2000).

[RB] Rey-Bellet, L.: Open classical systems. In S. Attal, A. Joye, and C.-A. Pillet, editors, Open Quantum Systems II: The Markovian Approach, volume 1881 of Lecture Notes in Mathematics. Springer, New York, (2006).

[RBT] Rey-Bellet L., Thomas, L.E.: Fluctuations of the entropy production in anharmonic chains. Ann. Henri Poincaré 3, 483 (2002). 